\title{
Microfabricated electrospray emitter arrays with integrated extractor and accelerator electrodes for the propulsion of small spacecraft
}

\author{
S Dandavino ${ }^{1}$, C Ataman ${ }^{1,3}$, C N Ryan ${ }^{2,4}$, S Chakraborty ${ }^{1}$, D Courtney ${ }^{1}$, \\ J P W Stark ${ }^{2}$ and $\mathrm{H}_{\text {Shea }}{ }^{1}$ \\ ${ }^{1}$ Institute of Microtechnology, Microsystems for Space Technologies Laboratory, EPFL, Neuchatel, \\ Switzerland \\ 2 School of Engineering and Materials Science, Queen Mary University of London, London, E1 4NS, UK \\ ${ }^{3}$ Department of Microsystems Engineering, University of Freiburg, Germany \\ ${ }^{4}$ Surrey Space Center, University of Surrey, Guilford, UK \\ E-mail: simon.dandavino@epfl.ch
}

Received 3 February 2014, revised 3 April 2014

Accepted for publication 29 April 2014

Published 5 June 2014

\begin{abstract}
Microfabricated electrospray thrusters could revolutionize the spacecraft industry by providing efficient propulsion capabilities to micro and nano satellites $(1-100 \mathrm{~kg})$. We present the modeling, design, fabrication and characterization of a new generation of devices, for the first time integrating in the fabrication process individual accelerator electrodes capable of focusing and accelerating the emitted sprays. Integrating these electrodes is a key milestone in the development of this technology; in addition to increasing the critical performance metrics of thrust, specific impulse and propulsive efficiency, the accelerators enable a number of new system features such as power tuning and thrust vectoring and balancing. Through microfabrication, we produced high density arrays $\left(213\right.$ emitters $\left.\mathrm{cm}^{-2}\right)$ of capillary emitters, assembling them at wafer-level with an extractor/accelerator electrode pair separated by micro-sandblasted glass. Through IV measurements, we could confirm that acceleration could be decoupled from the extraction of the spray-an important element towards the flexibility of this technology. We present the largest reported internally fed microfabricated arrays operation, with 127 emitters spraying in parallel, for a total beam of 10-30 $\mu \mathrm{A}$ composed by $95 \%$ of ions. Effective beam focusing was also demonstrated, with plume half-angles being reduced from approximately $30^{\circ}$ to $15^{\circ}$ with $2000 \mathrm{~V}$ acceleration. Based on these results, we predict, with $3000 \mathrm{~V}$ acceleration, thrust per emitter of $38.4 \mathrm{nN}$, specific impulse of $1103 \mathrm{~s}$ and a propulsive efficiency of $22 \%$ with $<1 \mathrm{~mW} /$ emitter power consumption.
\end{abstract}

Keywords: electrospray, spacecraft propulsion, wafer-level integration, small satellites

(Some figures may appear in colour only in the online journal)

\section{Introduction}

Launched as secondary payloads to conventional satellites, small spacecraft $(<100 \mathrm{~kg})$ provide low-risk, low-cost access to space. Combinedwith theincreasing capabilitiesand performance ofminiature sensors, actuators and electronics, they open up an exciting new branch of space exploration, accessible to universities and modest organizations. Demonstrating these possibilities, Surrey Satellite Technology $\operatorname{Ltd}^{5}$ and the NASA AMES research center 6 both launched in early 2013 (25 February and 21 April,

5 www.sstl.co.uk

${ }^{6} \mathrm{http}: / /$ phonesat.org 


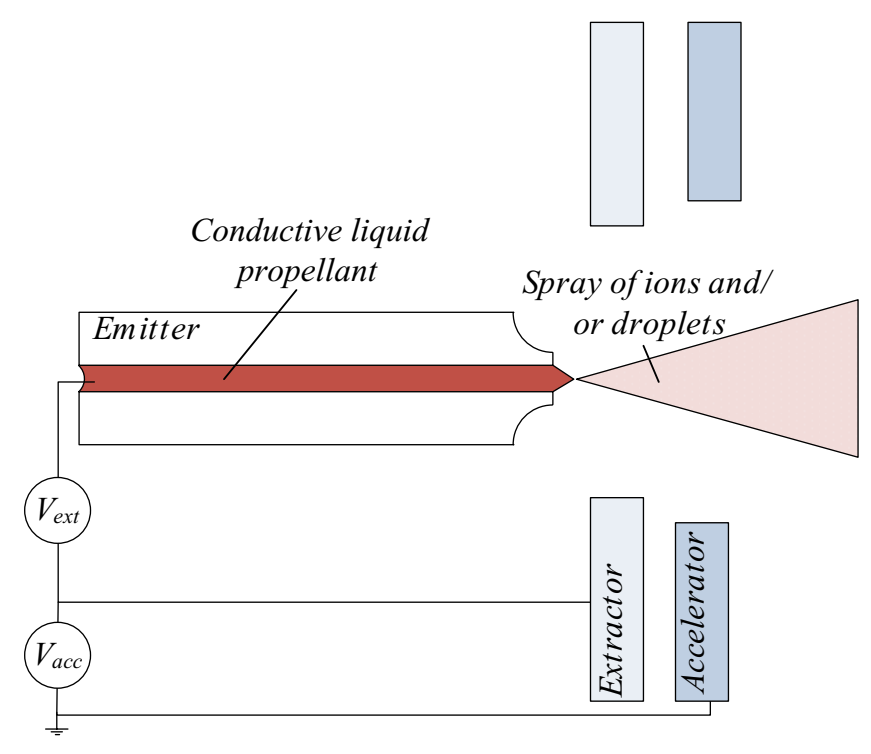

Figure 1. Basic operation of an electrospray thruster with an extraction and acceleration stage. A potential $V_{\text {ext }}$, positive or negative, is applied between the conductive liquid propellant and an extraction electrode aligned with the capillary emitter to generate the spray. An accelerator electrode can provide an additional potential $V_{\text {acc }}$ to increase the thruster performance.

respectively) miniature satellites driven by commercial offthe-shelf smartphones. Yet such missions remain limited by the current lack of a critical technology: a flexible and efficient miniaturized propulsion system capable of carrying the spacecraft beyond their initial orbit.

Electrospray thrusters are acknowledged as one of the most promising technologies to fill this gap, as demonstrated by their inclusion in NASA's Space Technology Roadmap for In-Space Propulsion Systems [1]. The electrostatically generated spray of high velocity particles creates thrust by direct momentum transfer with few sources of efficiency loss - a fundamental advantage of this technology. Several electrospray thruster systems are in or approaching commercialization [2, 3], yet none are microfabricated.

Microfabrication provides a natural evolution of the technology, building on its strengths by allowing the creation of highly integrated, compact systems ideal for miniature spacecraft. The small feature sizes are key to achieve the high fluidic impedance and thereby low flow rates required for high propellant usage efficiency (with the metric of specific impulse $\left.\left(I_{\mathrm{sp}}\right)\right)$, an advantage over conventional systems. Such small emitting features typically generate low thrust (T) levels (tens of $\mathrm{nN}$ ). However, microfabrication permits large arrays of high specific impulse emitters on a single die; critical to achieving useful (hundreds of $\mu \mathrm{N}$ ) thrusts at moderate voltages and within typical small satellite power budgets. The MicroThrust ${ }^{7}$ project was an European initiative aimed at developing a thruster module based on these microfabricated electrospray thrusters.

In electrosprays, a conductive liquid propellant (often an ionic liquid) is transported to an extraction site where a large electrical field is applied by precisely aligned electrodes. Increasing the electrical pressure, the liquid' surface tension can be overcome, deforming the liquid meniscus into a conic

7 www.microthrust.eu shape (Taylor cone [4]), which to a first order stabilizes with a half-angle of $49.3^{\circ}$. The sharpened liquid tip generates a large electric field concentration, in the order of $1 \mathrm{GV} \mathrm{m}^{-1}$, sufficient to atomize the liquid and generate a high velocity particle spray [5]. The extraction field is provided by a single electrode stage, and later stages can provide acceleration and focusing of the spray. We label the potentials supplied by each electrode as $V_{\text {ext }}$ and $V_{\text {acc }}$, as shown in figure 1.

Typically, electrospray emitters can be externally [6], porously [7] or internally [8,9] fed (figure 2). We use the internal approach with passive feeding, relying on capillarity to deliver the liquid. Passive feeding implies that no active pressure control is enforced and that the internal liquid pressure will be driven by the specific configuration of the emitters/ reservoir system. It is a system where the liquid is initially static and where flow is entirely induced and controlled by the applied electric field. In a complete system, we envision grooved reservoir walls and porous filling to deliver the liquid to the emitter. As long as the capillary pressure of the small emitters is sufficiently larger than that of these guiding features, the emitters should remain the driving factor, pulling propellant from the reservoir as they get depleted. In this way, we remove the need for pressurized tanks and lines-a great advantage from a system perspective. The present work does not include such a reservoir and we employ gravity to bring the propellant in contact with the emitters, delivering small amounts of liquid on the backside of the thruster. It is important to note that this pressure, due to gravity, is not sufficient to overcome the capillary holding pressure once filled. Hence a flow is only induced, and controlled, by the emission processes after applying a voltage.

Depending on the operating conditions and emitter design, the nature of the emitted species can be modified, from single ions to nanodroplets. In the purely ionic regime (PIR), the thruster boasts optimal specific impulse, though at the cost of low thrust per emitter. This tradeoff is highlighted in equations (1) and (2), where $g_{0}$ is the gravity constant, $I_{\mathrm{T}}$ is the emitted beam current, $V_{\mathrm{b}}$ the beam potential, $\frac{q}{m}$ is the charge over mass ratio of the emitted particles and $\dot{m}$ is the propellant flow rate.

$$
\begin{gathered}
T=I_{\mathrm{T}} \sqrt{2 V_{\mathrm{b}} \frac{m}{q}} \\
I_{\mathrm{sp}}=\frac{T}{\dot{m} g_{0}}=\frac{1}{g_{0}} \sqrt{2 V_{\mathrm{b}} \frac{q}{m}}
\end{gathered}
$$

An attractive configuration is to operate in PIR with arrays of emitters (thousands), effectively multiplying the thrust while retaining the high $I_{\text {sp. }}$.

Externally fed [10] and porous [11] emitters have been shown to operate principally in PIR using a variety of propellants. Internally fed emitters generate a plume often formed by a mix of both types of species, with the PIR much more difficult to reach. Romero-Sanz et al [12] achieved this in 2003 with the ionic liquid $\mathrm{EMI}-\mathrm{BF}_{4}$ and $20-40 \mu \mathrm{m}$ inner diameter needles. By actively controlling the pressure drop across the capillary emitter, they showed that PIR could be reached by enforcing very low flow rates, in the order of $20 \times 10^{-12} \mathrm{Kg} \mathrm{s}^{-1}$ for their configuration. An alternative means to reduce the propellant flow rate $\dot{m}$ 


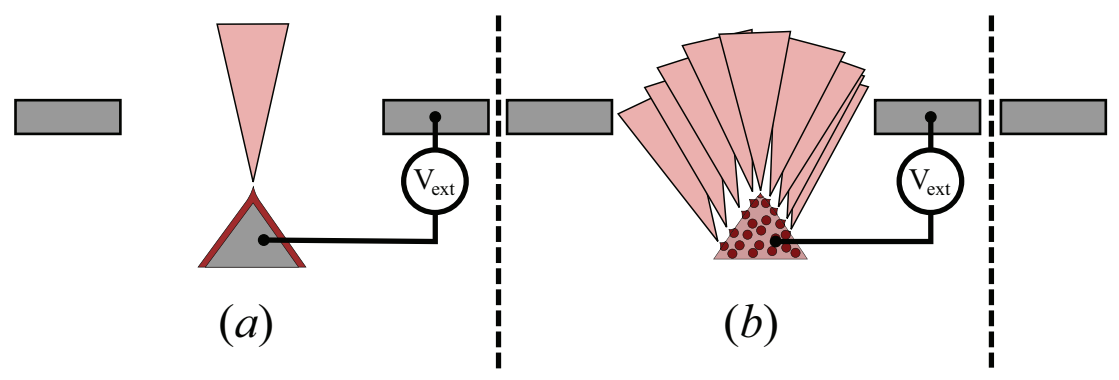

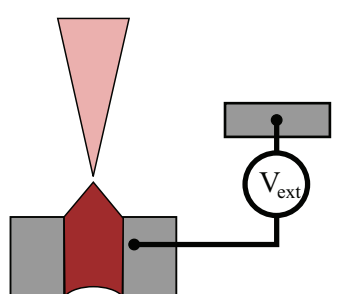

(c)

Figure 2. Common types of electrosprays. (a) Externally wetted: propellant is brought to the emission by wetting the surface of the emitter. (b) Porous: propellant fills the porous material and feeds the emission site by capillarity. (c) Internally fed: liquid is contained in a capillary (typically cylindrical or slit) and brought to the emission site passively by capillarity or through active pressure control.

is to increase the fluidic impedance of the emitters $(\dot{m}=\Delta P / Z$, where $Z$ is the fluidic impedance and $\Delta P$ is the pressure drop), which can be achieved through microfabrication. For microfabricated emitters, PIR could be reached by our group [13] and De La Mora's [9], in both cases using very high impedance, bead-filled micro-capillaries yet still with active pressure control.

PIR has never been achieved using passively fed, internally wetted emitters, as targeted by our recent work. An unpressurized and passive feeding system has the advantage of being more compact, since no high pressure reservoir or valve is needed. More importantly, it removes the risk of flooding due to over-pressurization. Reaching the necessary high fluidic impedance without requiring the bead filling step is also an advantage, as it improves the uniformity of the fluidic impedance. The devices presented in this paper could nearly be operated in PIR, with typical plumes composed of $95 \%$ ions (monomers and dimers) using the ionic liquid EMI- $\mathrm{BF}_{4}$ as propellant.

Previous thruster prototypes produced by our group were comprised of microfabricated arrays of 19 bead-filled capillary emitters $[13,14]$ with a single extraction stage. Our latest generation uses a simpler, improved emitter fabrication process and wafer-level bonding of the electrodes, which we had proposed earlier [15]. The new process permits the large scale fabrication of high aspect ratio capillaries with the smallest $<10 \mu \mathrm{m}$ inner diameters to date. For the first time, we report on the wafer-level integration of individual acceleration electrodes - a critical step in the development of this technology. The addition of accelerators increases simultaneously the specific impulse, thrust and system efficiency, and introduces new system features, such as power tuning and thrust vectoring and focusing. Integrating these electrodes at wafer-level, we can provide individual accelerators for each extractor/emitter pair with high density and excellent alignment. Individual accelerators are advantageous over the one accelerator/multiple emitter configuration, since each spray is accelerated uniformly and there are no edge effects. Uniformity and alignment of the acceleration field is important: errors may lead to off-axis spraying which elevates the risk of spraying onto the electrodes, reducing the propulsive efficiency and thruster lifetime. Furthermore, off-axis spray might also lead to errors in thrust vectoring, since any asymmetry in spray produces spurious lateral torque.

In sections 2 and 3 of this paper, we discuss the effect of these electrodes on the thruster performance through modeling and simulation. The overall design of the thrusters is described in section 4 and the process allowing the fabrication of large (127 emitters) and uniform arrays, is presented in section 5 . In section 6 , we study the performance of the accelerator electrodes through I-V curves, time-of-flight measurements and beam shape characterization, discussing the results in section 7 .

\section{Impact of acceleration electrode}

The propulsive efficiency $\eta_{\mathrm{T}}$ is defined as the ratio between the propulsive beam power $P_{\mathrm{b}}$ and the input electrical power $P_{\mathrm{e}}$ required to generate it ((3), where $\dot{m}$ is the mass flow rate, $I_{\mathrm{emi}}$ is the source current and $V_{\text {app }}$ is the applied potential, equal to $V_{\text {ext }}+V_{\text {acc }}$ which are shown in figure 1$)$.

$$
\eta_{\mathrm{T}}=\frac{P_{\mathrm{b}}}{P_{\mathrm{e}}}=\frac{\frac{T^{2}}{2 \dot{m}}}{I_{\mathrm{emi}} V_{\mathrm{app}}}=\eta_{\mathrm{P}} \eta_{E} \eta_{\theta}
$$

The polydispersive efficiency $\eta_{\mathrm{P}}$, the energy efficiency $\eta_{E}$ and the angular efficiency $\eta_{\theta}$ are, assuming sufficient physical clearance of the electrodes and low propellant evaporation, the principal sources of propulsive efficiency loss [16]. Equations (4) and (5) highlight how thrust and specific impulse can be increased by supplying additional electrical power to the thruster.

$$
\begin{gathered}
T=\sqrt{2 \eta_{\mathrm{T}} \dot{m} P_{\mathrm{e}}} \\
I_{\mathrm{sp}}=\frac{1}{g_{0}} \sqrt{\frac{2 \eta_{\mathrm{T}} P_{\mathrm{e}}}{\dot{m}}}
\end{gathered}
$$

Yet for a given thruster geometry, stable spray only occurs in a relatively small extraction voltage range, limiting the ability to boost performance by a direct increase of $V_{\text {ext }}$. The introduction of the acceleration electrode enables this feature, since the applied potential $V_{\text {app }}$ can now be increased while keeping $V_{\text {ext }}$ at an optimal level. Of course, the square root relationship between the performance increase and electrical input power indicates that the advantages of acceleration will diminish with increasing voltage, limiting the scale-up potential. Yet at the modest voltages of a few $\mathrm{kV}$, which can well be supplied by miniaturized power sources [17], the increase in thrust and $I_{\mathrm{sp}}$ can have a major impact on the mission design and feasibility-entirely justifying the additional power requirement. 


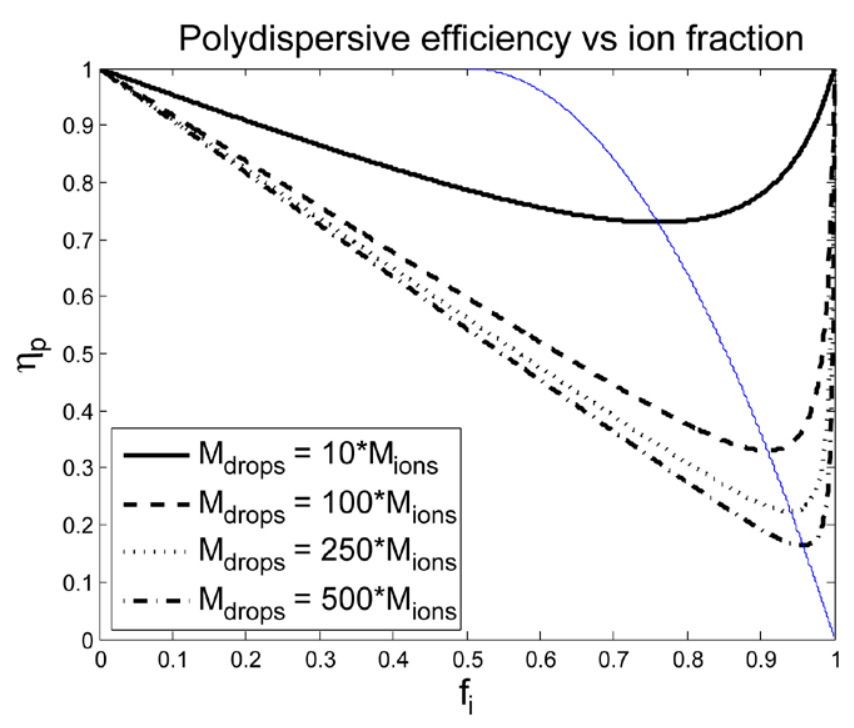

Figure 3. Polydispersive efficiency versus ion fraction for different droplet size. Larger droplets lead to a sharper decline in efficiency. Approaching PIR, small amounts of droplets quickly reduce the efficiency of the thruster. The blue line follows minimum efficiency points, corresponding to $f_{i}=\frac{1-\sqrt{\epsilon}}{1-\epsilon}$

Accelerator electrodes may also play a vital role in enabling long thruster lifetimes. Through decoupling thrust and current control $\left(I_{\mathrm{emi}}\right)$, this arrangement will ensure that variable thrust can be achieved without compromising charge balancing schemes necessary to suppress reported electrochemical degradation of the ionic liquid propellant [18].

Acceleration provides the additional advantage of increasing the propulsive efficiency, in particular in terms of the energy and the angular efficiencies.

The energy efficiency, given by (6) [19], relates the equivalent potential $V_{\mathrm{b}}$ that will be reached by an emitted particle going through an electric field provided by electrodes at potential $V_{\text {app. }}$

$$
\eta_{E}=\frac{\left|V_{\mathrm{b}}\right|}{\left|V_{\text {app }}\right|}=1-\frac{V_{\text {loss }}+|R I|}{\left|V_{\text {app }}\right|}
$$

In (6), $V_{\text {loss }}$ is related to the electrical energy required to form the cone and extract particles from the liquid while $R I$ is the potential drop due to ohmic losses in the liquid.

For a highly conductive ionic liquid, we approximate $R$ to 0 . $V_{\text {loss }}$ is not related to the post-acceleration and is constant regardless of the acceleration potential applied, such that the efficiency will approach unity as the acceleration voltage is increased. The value of $V_{\text {loss }}$ is difficult to predict and can be most easily measured through the use of retarding potential analysis (RPA). For this model, we use the value of $100 \mathrm{~V}$, as previously reported for this type of emitters, emitting in mixed droplet/ion mode [14]. In the PIR, energy losses below $15 \mathrm{eV}$ have been reported [20, 21], such that $100 \mathrm{eV}$ remains a conservative value.

For a uniform spray, the angular efficiency is given by (7) [19], where $\theta$ is the beam half-angle (BHA) and depends inversely on the acceleration voltage. The angular efficiency describes the momentum wasted by the off-axis component of the emitted particles.
Table 1. Breakdown of performance improvement with applied acceleration potential. We assume $V_{\mathrm{ext}}=1000 \mathrm{~V}$ and that the beam half-angle decreases linearly from $30^{\circ}$ to $20^{\circ}$ between 0 and $3000 \mathrm{~V}$ acceleration.

\begin{tabular}{lllllll}
\hline$V_{\text {acc }}$ & $\sqrt{\frac{V_{\text {appacc }}}{V_{\text {app } 0}}} \sqrt{\frac{\eta_{\text {Pacc }}}{\eta_{\mathrm{P} 0}}}$ & $\sqrt{\frac{\eta_{\text {Eacc }}}{\eta_{\mathrm{E} 0}}}$ & $\sqrt{\frac{\eta_{\theta \text { acc }}}{\eta_{\theta, 0}}}$ & $\sqrt{\frac{\eta_{\text {Tacc }}}{\eta_{\mathrm{T}, 0}}}$ & \\
\hline $0 \mathrm{~V}$ & 1.00 & 1.00 & 1.00 & 1.00 & 1.00 & 1.00 \\
$1000 \mathrm{~V}$ & 1.41 & 1.00 & 1.03 & 1.01 & 1.04 & 1.47 \\
$2000 \mathrm{~V}$ & 1.73 & 1.00 & 1.04 & 1.03 & 1.07 & 1.85 \\
$3000 \mathrm{~V}$ & 2.00 & 1.00 & 1.04 & 1.04 & 1.08 & 2.16 \\
\hline
\end{tabular}

$$
\eta_{\theta}=\cos ^{4}\left(\frac{\theta}{2}\right)
$$

Electrospray sources operating in the PIR have reported BHA in the order of $15^{\circ}$ to $20^{\circ}$ [22], although sources in mixed ion/ droplet mode have exhibited wider plumes of $30^{\circ}-40^{\circ}$ [23, 24].

It is also useful to discuss the polydispersive efficiency (8), which results from the extraction and acceleration of different particle species. For two distinct and discrete species, we write $\eta_{\mathrm{P}}$ as:

$$
\eta_{\mathrm{P}}=\frac{\left(1-(1-\sqrt{\epsilon}) f_{1}\right)^{2}}{1-(1-\epsilon) f_{1}}
$$

where $f_{1}$ is the fraction of the current carried by the smaller species (with larger $q / m$ ) and $\epsilon$ is the ratio of the charge-overmass ratios.

The polydispersive efficiency is not related to the acceleration voltage but to the composition of the spray as generated by the extraction electrode. Figure 3 plots $\eta_{\mathrm{P}}$ for different droplet masses. The larger the droplet size, the more quickly the efficiency drops off when ions are introduced. Although electrosprays operating in the droplet mode have been shown to display remarkable size homogeneity [25] and consequently large $\eta_{\mathrm{P}}$, the droplet mode inevitably leads to low specific impulse (equation (2)). To increase the $I_{\mathrm{sp}}$, the droplet size must be reduced, which often leads to a gradual increase in ion content and lower efficiency. Operation in the PIR, with little to no droplets (well below 1\%) present, offers a solution to this tradeoff. In this case, both high efficiency and specific impulse are achieved.

Combining the effect of increased beam potential and propulsive efficiency, and assuming that acceleration does not affect the propellant flow rate and emitted current, we can define an acceleration performance factor, $\kappa_{a}$, as the ratio between the thrusts of an accelerated and unaccelerated beam:

$$
\kappa_{a}=\frac{T_{\mathrm{acc}}}{T_{0}}=\frac{I_{\mathrm{sp} . \text { acc }}}{I_{\mathrm{sp} .0}}=\sqrt{\frac{V_{\text {appaccelerated }}}{V_{\text {app unaccelerated }}}} \sqrt{\frac{\eta_{\mathrm{T}_{\text {accelerated }}}}{\eta_{\mathrm{T}_{\text {unacelerated }}}}}
$$

Table 1 lists computed values of $\kappa_{a}$ and its constituents with applied acceleration ranging from 0 to $3000 \mathrm{~V}$. We assumed an extraction voltage $V_{\text {ext }}=1000 \mathrm{~V}$ and a linear decrease in the plume half angle so that the angle is decreased $30^{\circ}$ to $20^{\circ}$ between $0 \mathrm{~V}$ and $3000 \mathrm{~V}$ acceleration.

With the above assumptions, a 2.16 increase in thrust and $I_{\text {sp }}$ can be obtained with $3000 \mathrm{~V}$ acceleration, independently 


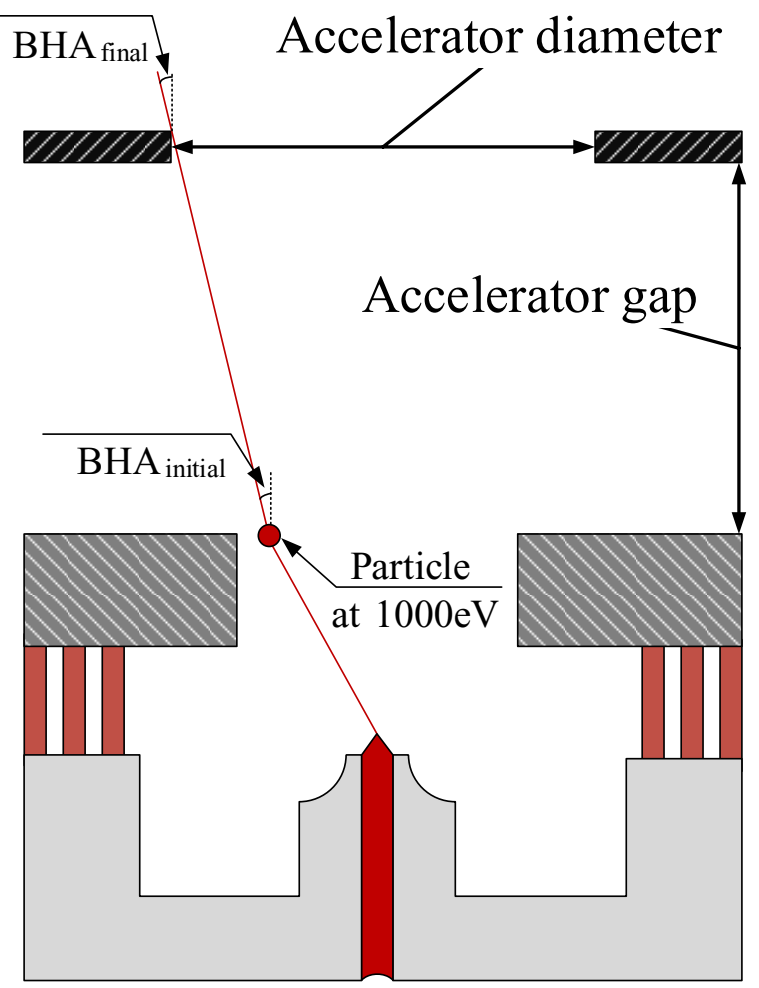

Figure 4. Cross-section diagram indicating key elements of the simulations. Parameters varied are the accelerator diameter and gap (spacing from the extractor electrode). For ion beam simulations, a particle is generated at the exit of the extractor with an energy of $1000 \mathrm{eV}$ and an initial direction $B H A_{\text {initial }}$. The direction of the particle is probed at the exit of the accelerator to determine how its trajectory was affected.

of beam composition and emitted current. This gain, however, comes at the cost of a $4 \mathrm{x}$ increase in electrical power.

To compute the efficiencies in table 1, we made two major assumptions. The first is that the acceleration did not have an affect on the nature or amount of charge being emitted (mass flow rate). Second is the beam half angle reduction from $30^{\circ}$ to $20^{\circ}$. In the next section, we will review these assumptions through simulations.

\section{Simulations}

\subsection{Extraction field and electrospray onset}

Modeling of the electrical field in the emission region was done using COMSOL, extending the model of Krpoun [26] to study the effect of the acceleration electrode on onset. The iterative procedure takes a $49.3^{\circ}$ truncated cone topped by a spherical tip. The cone is modeled as a solid, perfectly conductive surface which is gradually sharpened, keeping equilibrium between the liquid surface tension and electrical pressure. We compared the electrical field induced at the tip with and without accelerators, using a fixed potential of $1000 \mathrm{~V}$ between the emitter and extractor. For the accelerators, we varied two geometric parameters: the accelerator inner diameter, ranging from $150 \mu \mathrm{m}$ to $1000 \mu \mathrm{m}$ and the extractor/ accelerator separation, from $100 \mu \mathrm{m}$ to $1500 \mu \mathrm{m}$ (see figure 4).
In both cases, and with up to $3000 \mathrm{~V}$ acceleration applied, we obtained less than $5 \%$ variation in electrical field, negligible compared to the effects of the extractor design. This result indicates that the accelerator has little impact on the electrical field in the extraction region, supporting the argument that acceleration can be treated independently from emission.

\subsection{Ion-beam optics}

SIMION $^{8}$ was used to simulate the focusing effect of the accelerator. Conceptually, we assume that the particles are distributed uniformly and with equal energy in a cone of halfangle BHA. We generate a particle at the exit point of the extractor electrode. This particle has an energy of $1000 \mathrm{eV}$, representing the acceleration of a single charge through the $1000 \mathrm{~V}$ extraction field, and an initial angle $B H A_{\text {initial }}=28.7^{\circ}$. This angle was chosen so that the particle would not physically impact the acceleration electrode in any simulated configurations, allowing us to compare the effects of accelerator inner diameter and extractor-accelerator separation. Note that the nature of the species (ion or droplet) is irrelevant in this case, as only the particle energy $\left(q \cdot V_{\text {beam }}\right)$ affects the trajectory. The extractor electrode, $50 \mu \mathrm{m}$ thick and spaced $50 \mu \mathrm{m}$ from the capillary emitter has an inner diameter of $168 \mu \mathrm{m}$. We probe the particle direction at the exit of the accelerator $\left(B H A_{\text {final }}\right)$ and compare it with the angle at the exit of the extractor to get the relative focusing. Again, the accelerator inner diameter and the extractor/accelerator spacing were varied, respectively from $370 \mu \mathrm{m}$ to $1000 \mu \mathrm{m}$ and from $100 \mu \mathrm{m}$ to $400 \mu \mathrm{m}$.

For these two design parameters, figure 5 shows how the beam half angle is reduced when introducing acceleration. Typically, a 30-35\% reduction seems achievable with $3000 \mathrm{~V}$ acceleration voltage $\left(B H A_{\text {final }} \approx 19^{\circ}\right)$. The accelerator inner diameter has an inverse relationship with the final beam half angle, showing that smaller accelerators are more effective at focusing the beam. The accelerator-extractor spacing also has an effect on the focusing, with larger spacing being beneficial. In both cases, though, the dimensions have, at least in the range studied, almost negligible effects. Indeed, the extreme cases in all simulations, for the best and worse focusing, would lead to less than $1 \%$ variation in $\kappa_{a}$.

\section{Electrospray thruster design}

The thruster design is a progression over previously published devices [14], which included only the extraction stage. We now introduce an acceleration electrode, completely integrated in the fabrication process. From the above simulations, we find that the extractor and accelerator design can be de-coupled and optimized individually, with the criteria of performance, reliability and manufacturability.

The end device results from the wafer-level integration of an emitter and electrode wafer using a laminated thin film. This enables the optimisation of the emitters and electrodes separately, providing valuable flexibility in the design. The emitter

\footnotetext{
${ }^{8}$ http://simion.com
} 


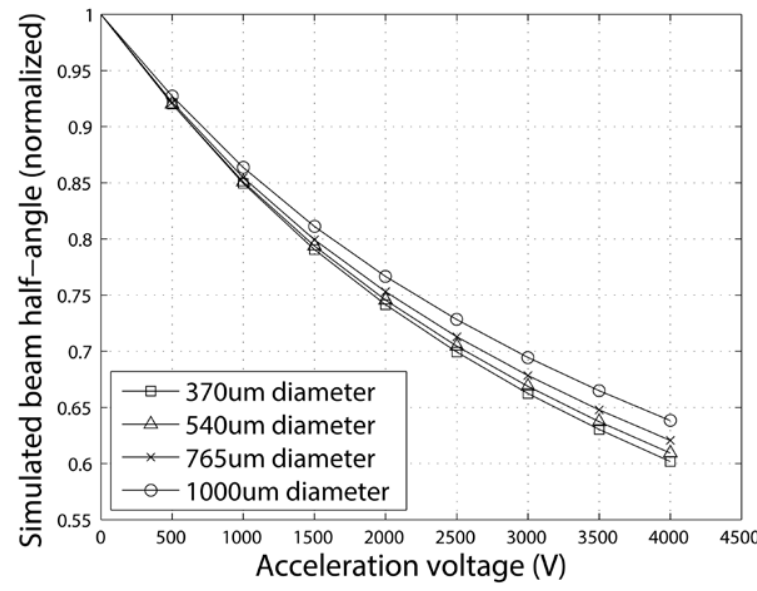

(a)

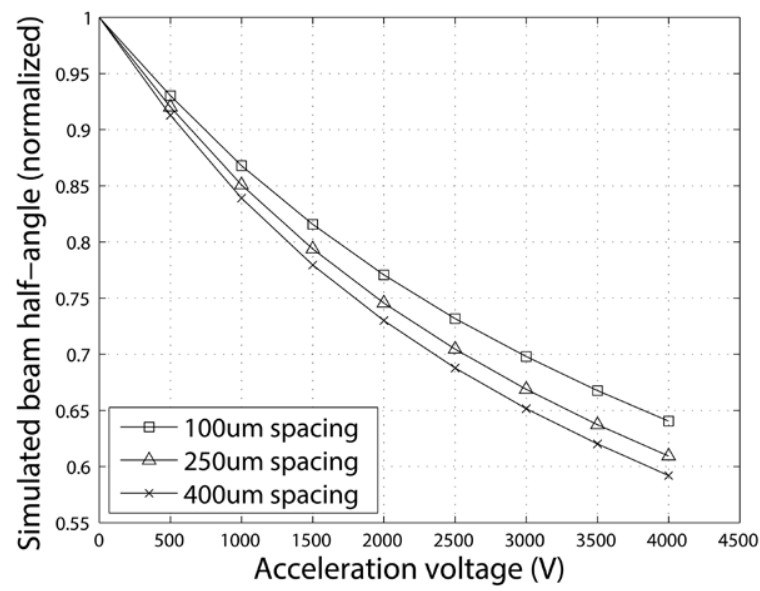

(b)

Figure 5. (a) Simulated beam half angle versus acceleration voltage for four accelerator inner diameters (extractor/accelerator spacing is set to $250 \mu \mathrm{m}$ ). (b) Simulated beam half angle versus acceleration voltage for three accelerator/extractor spacings (accelerator inner diameter is set to $540 \mu \mathrm{m}$ ). 30-35\% focusing is obtained, depending on the accelerator dimensions.

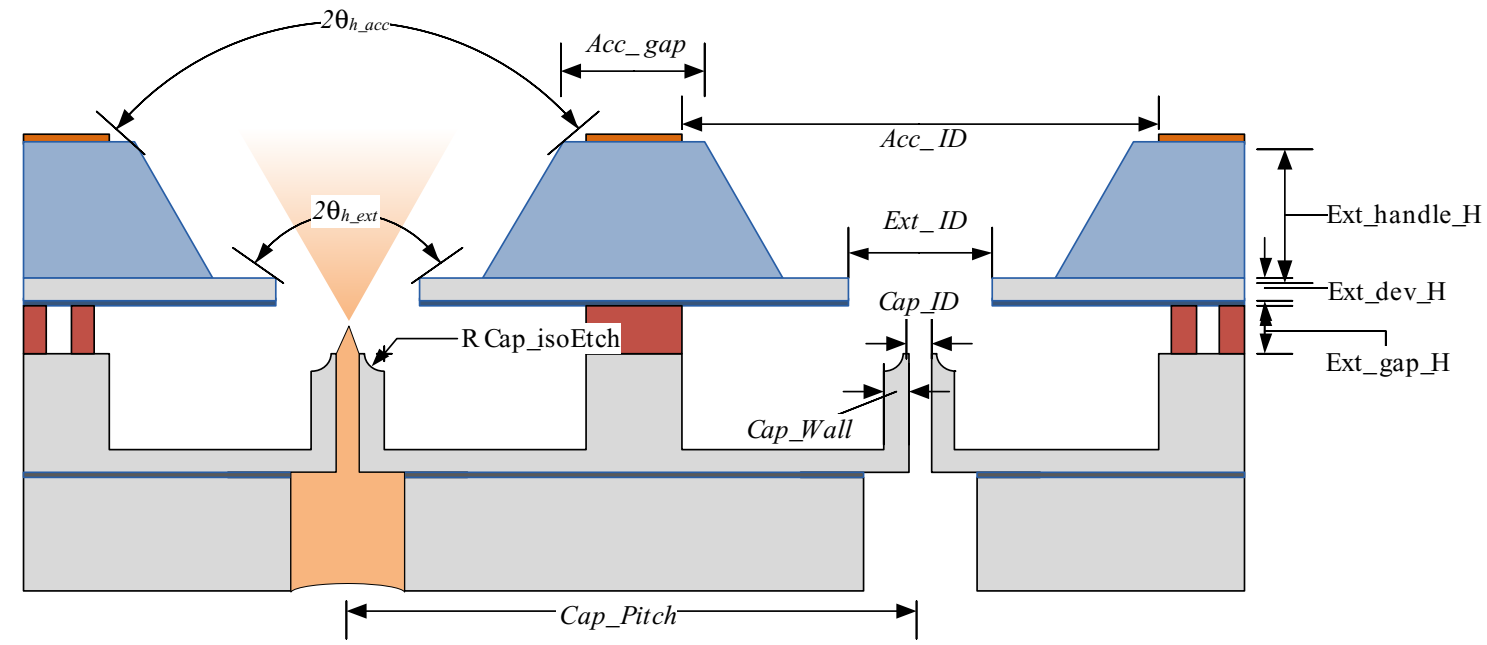

Figure 6. Cross-section diagram of thruster stack with key dimensions labeled.

wafer contains the capillary emitters and feedthrough reservoirs while the electrode wafer includes the extractor and accelerator electrodes. Figure 6 is a cross-section diagram of the thruster stack, with dimensions listed in table 2. With limited information on the real focusing effects of the accelerator electrode, we fabricated chips of three accelerator diameters, which allow increasing beam clearances. The baseline design allows approximately $40^{\circ}$ half-angle clearance, while the daring and safe variants allow $30^{\circ}$ and $50^{\circ}$ respectively. A smaller clearance, or accelerator diameter, is not only beneficial for its improved focusing effect, but most importantly to allow tighter array packing, which ultimately results in increased thrust density.

\subsection{Capillary emitter design}

A key dimension for the capillary emitter design is its inner diameter, $D$, which in turn determines its fluidic impedance. A high impedance will contribute to a low propellant flow rate which is critical to reaching the high $I_{\mathrm{sp}}$ PIR. Prior to this work, our devices relied on the filling of wider capillaries (24 $\mu \mathrm{m}$ inner diameter) with silica microbeads to tailor the hydraulic impedance and operation mode of the device [13], as also done by Lenguito et al [27]. However as our work increasingly focused on the fabrication of large arrays, this method became impractical due to the random arrangement of the beads which could lead to non-uniformities in operation. Instead, our group has focused on improving the etching step of the emitter interiors to allow narrower emitters with a more uniform impedance.

We aimed to achieve similar impedance to the bead-filled capillaries [13], which could be operated in PIR. A significant difference remains that these earlier emitters were operated with active pressure control and so it is difficult to guarantee that the new emitters, even with equal impedance would be equally ionic. Yet one can expect that a passively fed capillary operated in the same conditions and having no backpressure would necessarily have a flow rate lower or equal to a pressurized one, which would be consistent with PIR. 
Table 2. Design parameters of MicroThrust electrospray thrusters (All dimensions in micrometers.).

\begin{tabular}{llr}
\hline Parameter & Identification & $\begin{array}{l}\text { Nominal } \\
\text { dimension }(\mu \mathrm{m})\end{array}$ \\
\hline Emitter inner diameter & Cap ID & 5 \\
Emitter wall thickness & Cap Wall & 20 \\
Emitter pitch & Cap Pitch & $670,737,963$ \\
Emitter isotropic etch radius & Cap isoEtch & 15 \\
Extractor electrode diameter & Ext ID & 168 \\
Extractor electrode thickness & Ext dev H & 50 \\
Extractor-emitter gap & Ext gap H & 50 \\
Glass thickness & Ext handle H & 250 \\
Accelerator electrode & Acc ID & $370,540,765$ \\
$\quad$ diameter & & 150 \\
Accelerator to accelerator & Acc gap & \\
spacing & & \\
\hline
\end{tabular}

To compute the impedance of the bead-filled emitters, we follow a model adapted from Ergun [28], as suggested by Lenguito et al [9]. Using (10), we compute the effective inner diameter $D_{\text {eff }}$ of the emitter, which can be inserted in the Hagen-Poiseuille equation (11).

$$
\begin{gathered}
D_{\mathrm{eff}}=\sqrt{[4]} \frac{128}{600} \frac{D_{\mathrm{s}}^{2} D^{2} \epsilon^{3}}{(1-\epsilon)^{2}} \\
R_{\mathrm{fl}}=\frac{128 \mu L}{\pi D_{\mathrm{eff}}^{4}}
\end{gathered}
$$

where $\mu$ is the dynamic viscosity of the fluid (0.038 Pa. $s$ for $E M I-B F_{4}$ [29]), $L$ is the length of the capillary, $D_{\mathrm{s}}$ is the diameter of the microspheres and $D$ is the inner diameter of the capillary. The void fraction $\epsilon$, which ranges from 0 to 1 describes the packing of the beads inside the cylinder. For macroscopic cylindrical beds, $\epsilon$ stabilizes to 0.4 starting $5 D_{\text {s }}$ from the wall (both through model and experiment [30-32]). Closer to the wall, the packing varies considerably, with $\epsilon$ oscillating between approximately 0.25 and 0.65 . In our earlier bead filled devices, the cylinders themselves measured $24 \mu \mathrm{m}$ and the beads $5 \mu \mathrm{m}$, so that the 'stabilized' value of 0.4 cannot be directly used. Instead, we assume a range of $D_{\text {eff }}$ resulting from void fractions $\epsilon$ ranging from $0.25\left(D_{\text {eff }}=3.0 \mu \mathrm{m}\right)$ to 0.65 $\left(D_{\text {eff }}=9.1 \mu \mathrm{m}\right)$. Figure 7 shows how the bead filled emitters compare with the emitters presented in this work. We aimed for emitter inner diameters of $5.0+/-0.5 \mu \mathrm{m}$, but lateral attack in the etch process led to slightly wider fabricated devices $(7.9+/-0.5 \mu \mathrm{m})$, as will be discussed later. Considered in the coordinates of figure 11, our targeted impedance was therefore comparable to the low range predicted using bead filling. For our fabricated devices we estimate an order of magnitude lower impedance than our target and the high range of the bead-filled emitters.

The emitter tip is also sharpened using an isotropic etch which attacks the perimeter of the cylindrical emitter. This etch effectively reduces the width of the annular plateau at the tip of the capillary. This sharpening reduces the operation voltage and is favorable for liquid containment at the tip. We aim for $15 \mu \mathrm{m}$ lateral etch, leading to a $5 \mu \mathrm{m}$ plateau. This value is the maximum that can be safely achieved without risk of breaching the

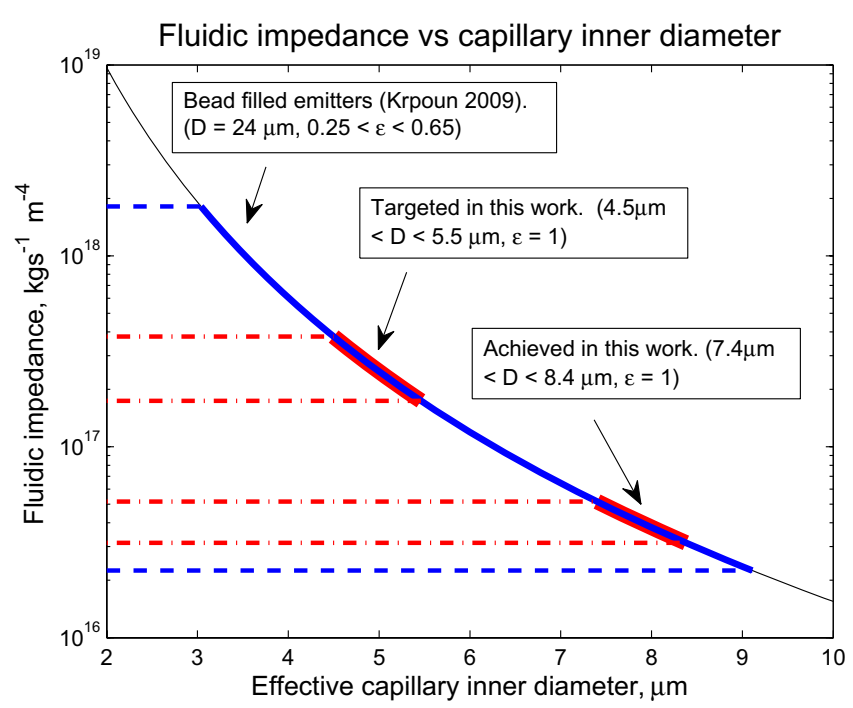

Figure 7. Estimated fluidic impedance of capillary emitters.

capillary tube considering the fabrication tolerances in terms of emitter inner and outer diameter as well as alignment.

\subsection{Electrode design}

A driving requirement is to minimize the extraction voltage. While a higher $V_{\text {ext }}$ may be useful to increase the beam energy and thruster performance, we find that a lower voltage at this level leads to a more reliable chip and simpler system design. Post-acceleration remains the preferred choice to increase the performance. From earlier simulations [26], we see that the key drivers to reduce $V_{\text {ext }}$ are the extractor electrode diameter and the extractor-emitter gap. The former is designed at $168 \mu \mathrm{m}$, enough to allow $40^{\circ}$ beam half-angle clearance while the latter is kept at $50 \mu \mathrm{m}$. Earlier devices used a $15 \mu \mathrm{m}$ film to separate the emitter and extractor, but this provided insufficient electrical insulation. Although the bulk film's breakdown strength was measured at $2.3 \mathrm{kV}$, leakage current problems forced the use of a thicker, $50 \mu \mathrm{m}$ film. Experiments showed that the leakage occurred at the edge of the die where conductive silicon dust generated at dicing contaminated the film. Insulation between the extractor and accelerator electrode levels is provided by $250 \mu \mathrm{m}$ of glass. This dimension should be as small as possible as it drives, given the non-zero beam half-angle, the packing density of the emitters. From the ion beam simulations of section 3 , we also see that this thickness, which forms the gap between extractor and accelerator, has minimal impact on beam focusing.

Thinner glass insulation may have been sufficient but led to low fabrication yield due to the difficult handling of thin glass wafers. The current thickness, expected to insulate upwards of $15 \mathrm{kV}$, allows significant flexibility in the system, effectively permitting any acceleration voltage to be applied within the limitations of typical small spacecraft. The opening of the glass on the top side, follows the allowed beam half-angle clearance and has diameter of $370 \mu \mathrm{m}, 540 \mu \mathrm{m}$ or $765 \mu \mathrm{m}$. The final baseline thruster includes 127 emitters packed with a density of 213 emitters $\mathrm{cm}^{-2}$. Variants have 91 (125 emitters $\mathrm{cm}^{-2}$ ) and 167 emitters $\left(257\right.$ emitters $\mathrm{cm}^{-2}$ ). 

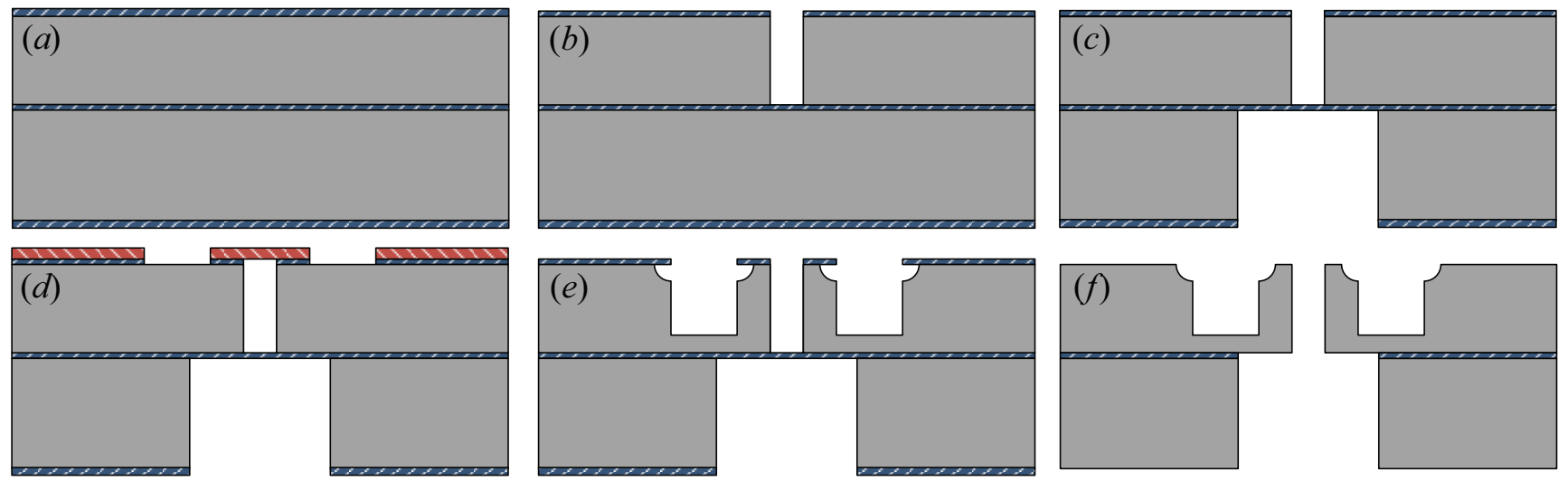

Silicon

Oxide

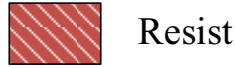

Figure 8. Fabrication steps for the emitter wafers. (a) The SOI wafers have $100 \mu \mathrm{m}$ thick device layer, $2 \mu \mathrm{m}$ thick buried oxide layer, and $500 \mu \mathrm{m}$ thick handle layer. With DRIE, first the capillary emitter interiors $(b)$ and the backside reservoir is patterned $(c)$. Thick resist is spun on the frontside and patterned to define the emitter exteriors $(d)$, which are etched in two steps $(e)$ before the buried oxide is removed by vapor $\mathrm{HF}(f)$.
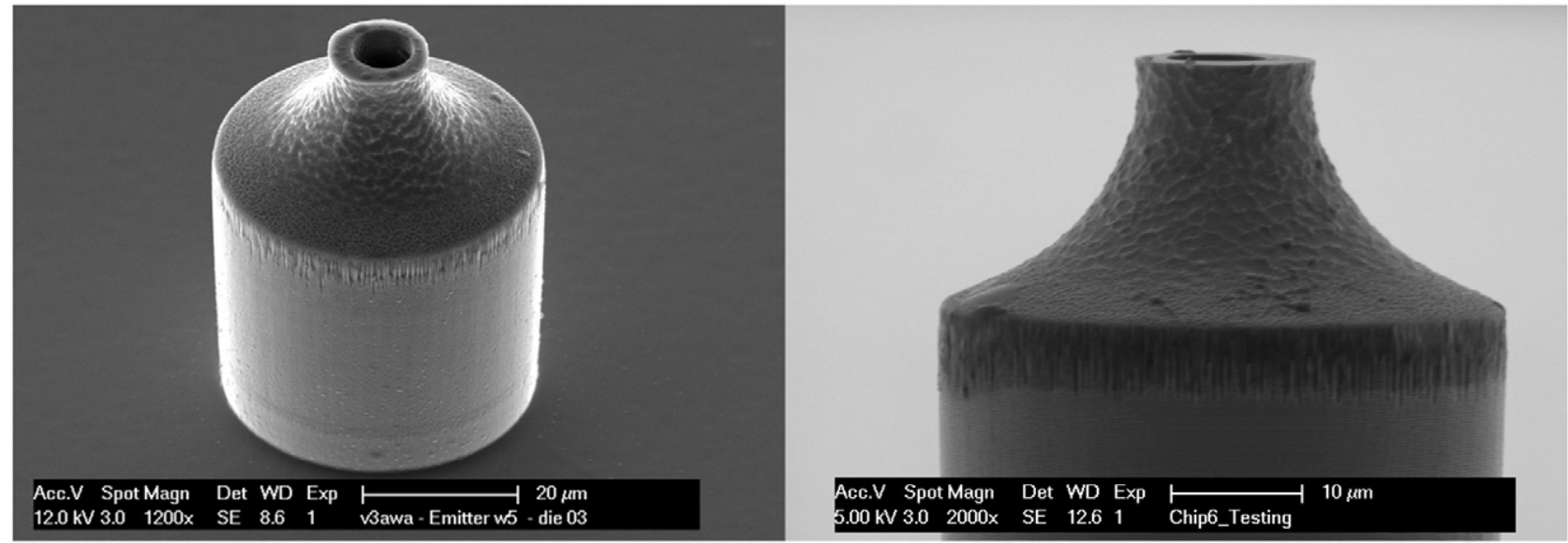

Figure 9. SEM images of single electrospray capillary emitters, highlighting the sharped tip of the emitter. The emitter interiors are typically measured at $7.9 \pm 0.5 \mu \mathrm{m}$.

\section{Thruster fabrication}

Although the end structure is complex, microfabrication and wafer-level integration techniques allowed decoupling of many structural complexities. In this particular case, the fabricated capillaries and integrated extractor/accelerator electrodes are fabricated on separate substrates, respectively the emitter wafer and electrode wafers.

\subsection{Capillary emitter wafer}

Principal fabrication steps of the emitter wafer are shown in figure 8. A $2.2 \mu \mathrm{m}$ thermal oxide layer is grown on the starting SOI wafer $(100 / 2 / 350 \mu \mathrm{m})(a)$. The oxide layer is patterned using a $4.8 \mu \mathrm{m}$ thick 10:1 AZ9260:PGMEA mixture photoresist spun at $3500 \mathrm{rpm}$. The emitter interior is then patterned by DRIE (b). Control of this etch is critical as it will drive the fluidic impedance of the emitter. Fabricated emitters have an inner diameter measured at $7.9 \pm 0.5 \mu \mathrm{m}$, a result of the slight widening typically seen in high aspect ratio DRIE. Next, the liquid reservoir is patterned by DRIE on the backside of the wafer, stopping on the buried oxide $(c)$. Third, the exterior of the emitters is patterned using the same resist mixture described above $(c)$. The resist exhibits sufficient viscosity to cover the emitter orifices cleanly and provide a uniform surface. In some cases, resist residues remained inside the emitters, even after several attempts at plasma $\mathrm{O}_{2}$ cleaning, indicating that further optimization is required at this stage. The resist and oxide hard mask are then patterned before a final, twostep dry etch is performed. The first step, isotropic, sharpens the emitter tip, reducing the onset voltage and restricting the ionic liquid from overflow. The second step is anisotropic and provides clearance from the emitter tip to the silicon bulk $((d)-(e)$ ). A final vapor $\mathrm{HF}$ etch is done to remove the BOX, connecting the backside reservoir to the capillary emitter ( $f$ ). SEM images of completed single emitters (top side) are seen in figure 9.

\subsection{Extraction and acceleration electrode wafer}

The novel electrode process provides each emitter with an individual extractor and, for the first time, accelerator electrode. 


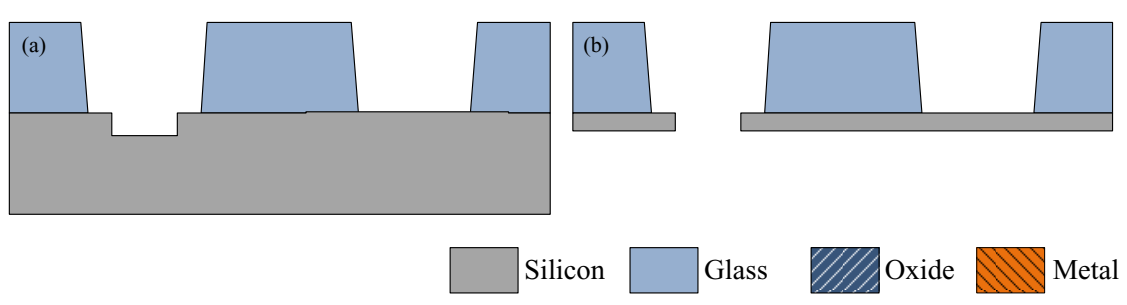

Figure 10. Fabrication process of the electrode wafer. (a) The patterned glass, $250 \mu \mathrm{m}$ thick, is patterned by micro sandblasting before anodic bonding to a patterned silicon wafer. The wafer is ground and polished $(b)$ before oxide and metal are deposited on the bottom and top, respectively $(c)$.

The process begins with the anodic bonding of a $300 \mu \mathrm{m}$ thick silicon wafer to a $250 \mu \mathrm{m}$ thick Borofloat 33 wafer patterned by micro sandblasting (figure $10(a)$ ). With its low thermal expansion coefficient $\left(3.25 \cdot 10^{-6} \mathrm{~K}^{-1}\right)$, Borofloat 33 is a viable alternative to Pyrex for anodic bonding.

The silicon wafer is patterned through a single DRIE, while the glass is patterned by a novel micro-sandblasting method [33] offered by Icoflex 9 .

Unlike with silicon, deep high-aspect ratio patterning of glass is challenging. While laser machining allows precise structuring, it is time consuming and expensive. Photopatternable glasses (PPGs) such as APEX ${ }^{10}$ and Foturan ${ }^{11}$ offer good alternatives but are relatively difficult to process and even more difficult to be used in anodic bonding, due to their high thermal mismatch with silicon. HF based wet etches [34] are fast and well developed, but their isotropic nature limits the packing density that can be achieved. Micro sandblasting, on the other hand, is a fast and inexpensive wafer-level technique. Although limited aspect ratios can be achieved with this technique (of the order of $1: 1$ ) and despite being less precise than PPGs, it offers reasonable side-wall angles $\left(15-20^{\circ}\right)$ and is well suited for this application (See also Lenard et al [35]).

After bonding, the silicon is ground and polished to a thickness of $50 \mu \mathrm{m}$ to reveal the extractor pattern and create through-wafer openings. $2 \mu \mathrm{m}$ silicon dioxide is then sputtered on the bottom of the wafer to provide electrical insulation between the extractor and emitter levels, and metal is evaporated on the front side through a shadow mask to create the accelerator electrodes $(c)$. Due to the insular nature of the patterns to be masked, two depositions (200 nm thickness) are required to generate the adequate electrode pattern, each rotated by $180^{\circ}$. An SEM image of a cleaved electrode, before metal deposition, is shown in figure 11.

\subsection{Wafer-level integration}

In a final microfabrication step, the emitter and electrodes are wafer-level bonded using a dry photo-patternable negative resist (DuPont MX5050). The resist is laminated on the bottom of the electrode wafer and patterned by standard lithography and development. Alignment of the two wafers is done in a Suss Microtech MA6/BA6 in bond alignment configuration and the stack bonded by thermo-compression at $150^{\circ} \mathrm{C}$ and $0.3 \mathrm{MPa}$ in a Suss Substrate Bonder SB6.

\footnotetext{
9 www.icoflex.com

${ }^{10}$ www.lifebiosciences.com

11 www.schott.com
}

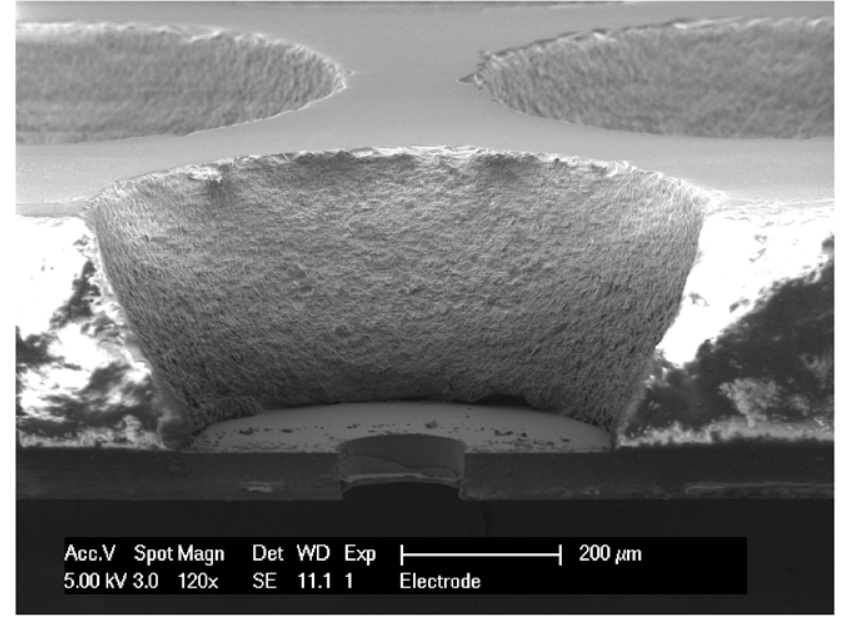

Figure 11. SEM cross-section image of micro-sandblasted glass insulator anodically bonded to extractor electrode.

Final alignment is typically better than $10 \mu \mathrm{m}$. Figure 12 shows SEM images of the assembled thruster stack while figure 13 is an optical image of a finished die.

\section{Test and characterization}

The test setup (figure 14) consists of two chambers: a main chamber for spraying and a second for the storage of the ionic liquid. The liquid is itself transferred using a glass capillary by applying a pressure differential and dropped on the backside of the assembly, where capillarity transports it to the emitter tip. The spray direction is vertically downwards.

The thruster chip is held within a simple holder, as illustrated in figure 15.

Electrical connections to the extractor and accelerator are made from the front side while connection to the emitter is through the ionic liquid, via a central metal section within the PEEK holder (not shown). Generally, the accelerator is grounded, while both extractor and emitter potentials are raised above ground to generate extraction and/or acceleration. The ionic liquid 1-ethyl3-methylimidazolium tetrafluoroborate $\left(\mathrm{EMI}-\mathrm{BF}_{4}\right)$ is used as the propellant. This ionic liquid is commonly used to demonstrate thrust and has been known to operate an a variety of regimes: purely ionic [11], mixed droplet/ion [3] or droplet.

The emitted spray is collected either by a large $(220 \mathrm{~mm})$ plate situated $500 \mathrm{~mm}$ downstream or by a small Kimball Physics Faraday cup mounted on a $280 \mathrm{~mm}$ translation stage at the same distance. The stage can move laterally on one axis, 


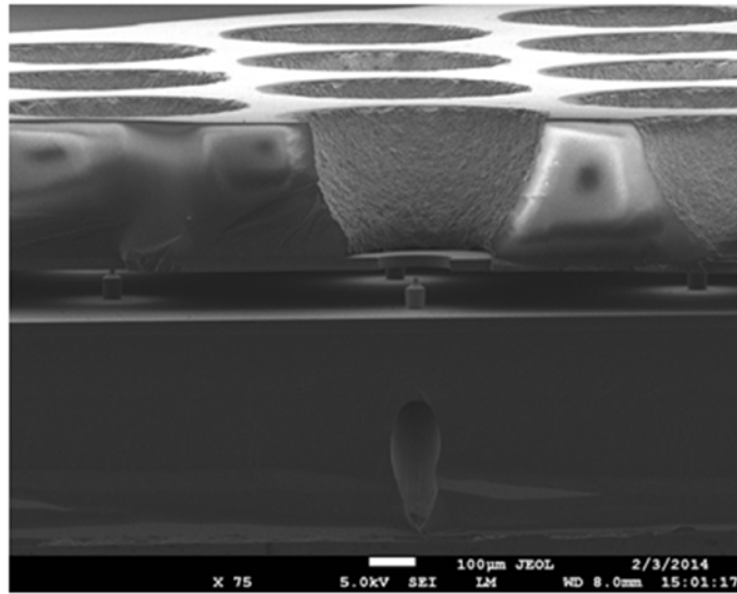

(a)

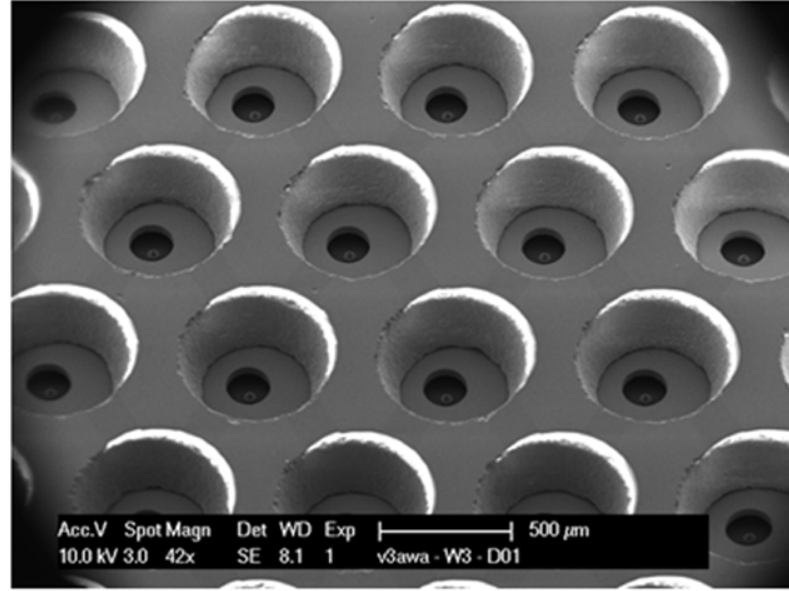

(b)

Figure 12. SEM images of thruster chips showing arrays of emitters aligned to individual extractor and accelerator electrodes.

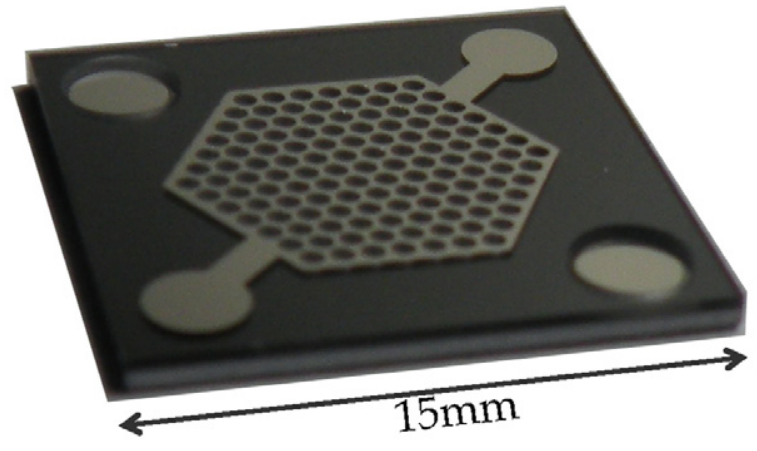

Figure 13. Optical image of diced thruster chip. The top right and bottom left metal pads allow contact with the accelerator layer, with the top left and bottom right contact the extractor level.

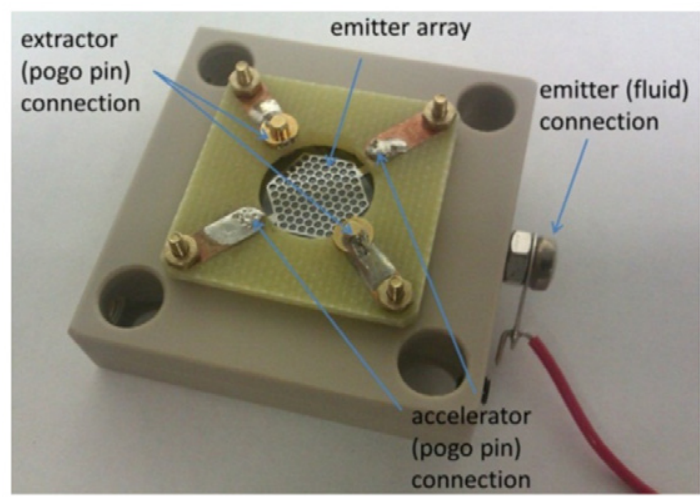

Figure 14. The test setup. For beam shape measurements, the large $(220 \mathrm{~mm})$ target plate is replaced by a small plate mounted on a small translation stage.

mapping the plume which is assumed to be axisymmetric. All the emitters of the array are contained within a $6 \mathrm{~mm}$ radius of the center, so that the effect of their finite position on the measured beam profile is negligible. The first configuration is used for time-of-flight (ToF) and IV measurements, while the second allows characterization of the plume shape. For the

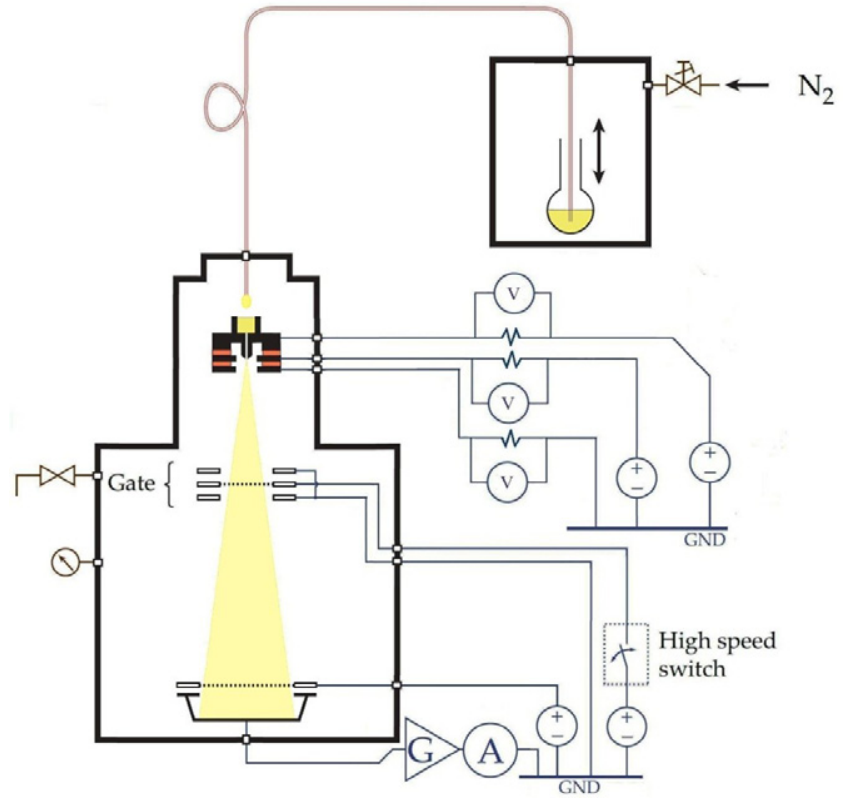

Figure 15. Emitter array installed in test assembly. Contact to the extractor and accelerator electrodes can be verified in situ by measuring the resistance between two opposite pins.

collection of ToF data, a DEI PVX-4150 high speed switch is used to rapidly ( $20 \mathrm{~ns}$ ) turn off spraying by connecting the extractor to the emitter potential. Monitoring the flight time of the particles, it is possible to determine their nature and consequently the generated thrust, specific impulse and propulsive efficiency [36].

In all cases, the current is measured on the emitter, extractor and accelerator by the measurement of the voltage drop across a resistor. More details on the test setup, in addition to extended characterisation of the thruster mounted in its breadboard assembly, can be found in [36].

Figure 16 plots the current measured at the emitter using the potential divider system against the applied voltage difference between the emitters and the extractor $\left(V_{\text {ext }}\right)$. The emitted current is proportional to the plume current. Data 


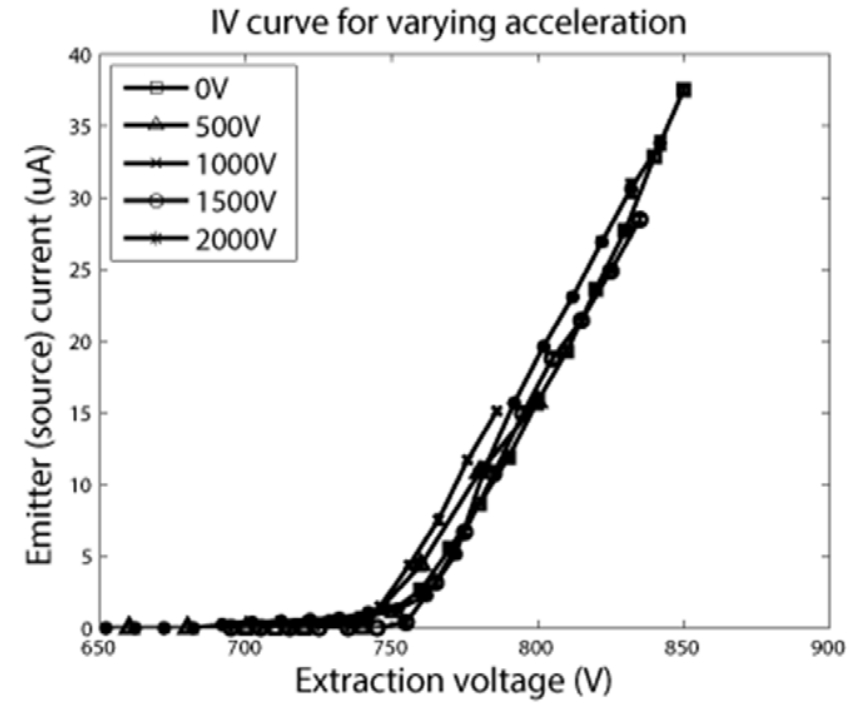

Figure 16. IV curve of 127 emitter array, showing the source current as a function of extraction voltage. Several curves, at different acceleration potentials, are plotted. The plot highlights the independence between the IV characteristics and the acceleration voltage.

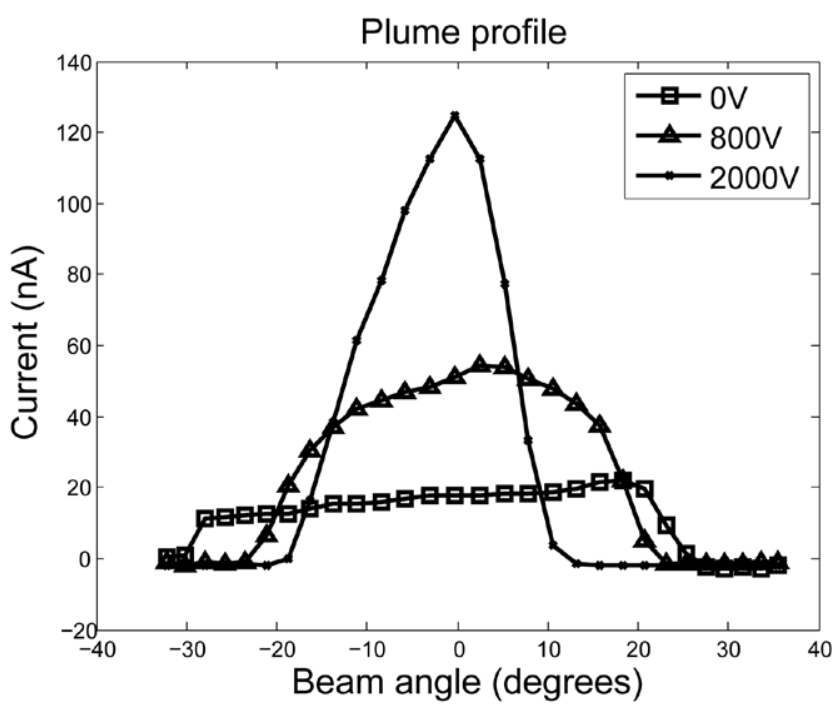

Figure 17. Example of plume profile with varying acceleration. As the acceleration is increased, the width of the beam decreases while the amount of current collected close to the center is increased.

is plotted for various acceleration voltages $\left(V_{\text {acc }}\right)$ defined as the voltage applied to the extractor to raise it above ground. The actual accelerator on the THC is at ground throughout. Data is shown for the baseline design, i.e. with $540 \mu \mathrm{m}$ accelerator diameter and 127 emitters. There is a strong linear trend of emitter current, independent of the acceleration voltage. This independence demonstrates that the electric field within the emitter-extractor region is not affected by field conditions downstream, as predicted by the onset voltage simulations. The gradient of the IV relationship in figure 16 is $\sim 0.4 \mu \mathrm{A}$ per emitter-extractor volt. This strong relationship is in agreement with previous studies using large colloid thruster arrays [7].

Figure 17 is an example of the plume profile from a single emitter as the applied acceleration voltage is increased from 0

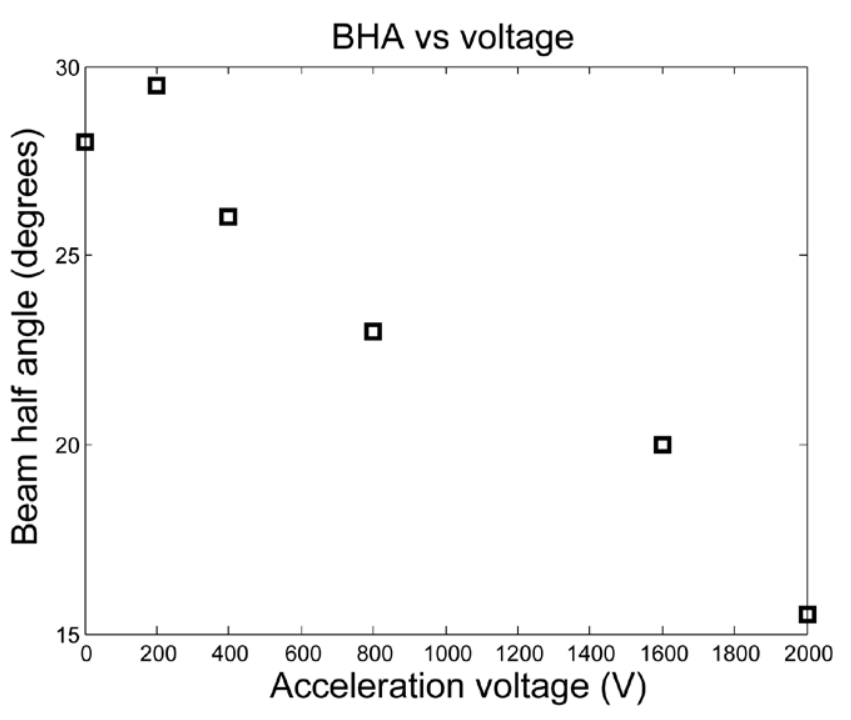

Figure 18. Beam half-angle versus acceleration voltage.

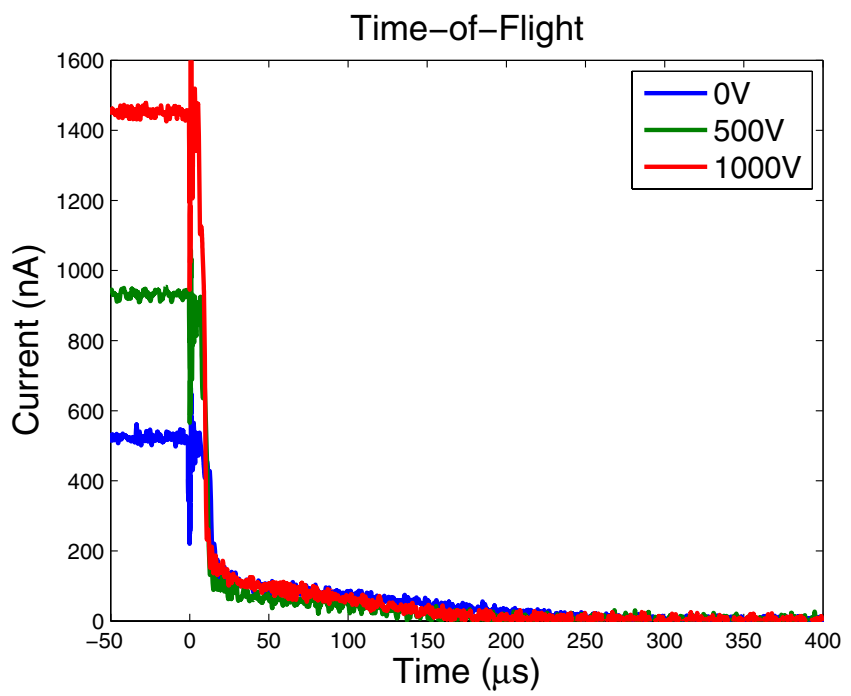

Figure 19. Time-of-flight curve taken with three accelerating voltages. The increased focusing due to acceleration enables the capture of increasingly larger portions of the beam, as reflected by the higher starting current.

to $2000 \mathrm{~V}$. A clear focusing effect is seen, with the plume profile reduced form $\sim 30^{\circ}$ to $\sim 15^{\circ}$, corresponding to an angular efficiency increase from $87 \%$ to $97 \%$. Figure 18 plots the measured BHA against acceleration voltage.

We use time of flight measurements to determine the nature of the emitted species and calculate the specific impulse and thrust. A sample ToF spectra is presented in figure 19. The plot displays a large initial drop corresponding to the arrival of ions followed by a quickly decreasing droplet tail. Typical results of 127 emitter arrays show that the beam is composed at $95 \%$ of ions (monomers and dimers) with the remaining droplets being clusters of $\approx 230$ ions. Table 3 summarizes these results and lists, for $850 \mathrm{~V}$ extraction, the resulting calculated flow rates, efficiency, specific impulse, thrust and power consumption. All values have been been divided by the array size and are consequently average values per emitter. 
Table 3. Measured beam composition using time of flight analysis.

\begin{tabular}{|c|c|c|c|c|c|c|c|c|c|c|}
\hline \multirow[b]{2}{*}{$\begin{array}{l}I_{\mathrm{emi}} \\
(\mathrm{nA})\end{array}$} & \multicolumn{2}{|c|}{ Species 1} & \multicolumn{2}{|c|}{ Species 2} & \multirow[b]{2}{*}{$\epsilon$} & \multirow[b]{2}{*}{$\begin{array}{l}\dot{m} \\
\left(\mathrm{~kg} \mathrm{~s}^{-1}\right)\end{array}$} & \multirow[b]{2}{*}{$\eta_{T}$} & \multirow[b]{2}{*}{$\begin{array}{l}I_{\mathrm{sp}} \\
(\mathrm{s})\end{array}$} & \multirow[b]{2}{*}{$\begin{array}{l}T \\
(\mathrm{nN})\end{array}$} & \multirow[b]{2}{*}{$\begin{array}{l}P \\
(\mu \mathrm{W})\end{array}$} \\
\hline & $\begin{array}{l}(q / m)_{1} \\
\left(\mathrm{C} \mathrm{kg}^{-1}\right)\end{array}$ & $f_{1}$ & $\begin{array}{l}(q / m)_{2} \\
\left(\mathrm{C} \mathrm{kg}^{-1}\right)\end{array}$ & $f_{2}$ & & & & & & \\
\hline 250 & $8.68 \mathrm{e} 5$ & 0.95 & $3.81 \mathrm{e} 3$ & 0.05 & $4.4 \mathrm{e}-3$ & $3.55 \mathrm{e}-12$ & 0.18 & 474 & 16.5 & 212.5 \\
\hline
\end{tabular}

Table 4. Expected thruster performance improvement with acceleration. All values are given per emitter.

\begin{tabular}{|c|c|c|c|c|c|c|c|c|}
\hline \multirow[b]{2}{*}{ Spray composition } & \multicolumn{4}{|c|}{ Unaccelerated } & \multicolumn{4}{|c|}{$3000 \mathrm{~V}$ Acceleration } \\
\hline & $\mathrm{T}(\mathrm{nN})$ & Isp (s) & $\eta_{\mathrm{T}}$ & $\mathrm{P}(\mu \mathrm{W})$ & $\mathrm{T}(\mathrm{nN})$ & Isp (s) & $\eta_{\mathrm{T}}$ & $\mathrm{P}(\mu \mathrm{W})$ \\
\hline Case $1^{\mathrm{a}}$ & 12.9 & 2421 & 0.72 & 212.5 & 30.1 & 5628 & 0.86 & 962.5 \\
\hline Case $2^{\text {b }}$ & 12.0 & 2550 & 0.71 & 212.5 & 28.0 & 5928 & 0.85 & 962.5 \\
\hline Case $3^{\mathrm{c}}$ (Observed) & 16.5 & 474 & 0.18 & 212.5 & 38.4 & 1103 & 0.22 & 962.5 \\
\hline
\end{tabular}

${ }^{\text {a }}$ Positive beam composed of $50 \%[\mathrm{EMI}]^{+}$and $50 \%\left[\mathrm{EMI}^{-\mathrm{BF}} 4\right]_{4}[\mathrm{EMI}]^{+}$

${ }^{b}$ Negative beam composed of $50 \%\left[\mathrm{BF}_{4}\right]^{-}$and $50 \%$ [EMI-BF $]\left[\mathrm{BF}_{4}\right]^{-}$

' Typical mix found when operating in 'mixed mode' with present emitters

\section{Discussion}

Characterization of the emitter arrays has shown very promising results in terms of the usability of acceleration electrodes. It was successfully shown that the acceleration and extraction stages could be decoupled, an important element to simplify the system and mission design. Beam shape focusing proved better than expected from simulations, with measured beam half-angles being focused from approximately $30^{\circ}$ to $15^{\circ}$ with $2000 \mathrm{~V}$ acceleration.

The composition of the beam, with $95 \%$ ions, is approaching the desired PIR, although the small amount of droplets drastically reduced the achieved specific impulse and efficiency. With the droplet size seen here, the minimum theoretical polydispersive efficiency occurs at $94 \%$, close to the present situation. Yet it is encouraging that the curve minimum (figure 3) was passed and that any further increase in the ion content will rapidly lead to better performance. We expect that this can be achieved by further reducing the inner diameters of the emitters, thereby increasing their hydraulic impedance and reducing the flow rate.

We can compare the performance of acceleration for the beam composition seen here with positive and negative ionic regimes. Table 4 lists the calculated thrust, specific impulse, total efficiency and power for unaccelerated and accelerated beams. Cases 1 and 2 describe, for comparison purposes, calculated performance parameters based on the current levels and focusing observed. Case 1 refers to a $50 \% / 50 \%$ mix of positive monomers and dimers while case two is the equivalent mix of negative monomers and dimers. Case 3 corresponds to the composition of a typically observed beam (95\% ions, with $\left.M_{\text {droplet }}=230 M_{\text {ion }}\right)$. In all cases, we use $V_{\text {ext }}=850 \mathrm{~V}$, $I_{\mathrm{emi}}=250 \mathrm{nA}$ and assume $30^{\circ}$ to $15^{\circ}$ focusing.

To illustrate the potential benefits of acceleration, we take as a reference a miniature HV power supply [17] developed for this application and capable of delivering $3800 \mathrm{~V}$ in the positive and negative polarities. With $\approx 800 \mathrm{~V}$ required for emission, we can use up to $3000 \mathrm{~V}$ acceleration, which increases all metrics by a factor of $2.32 \mathrm{x}$ over the unaccelerated case, at the cost of a $4.5 \mathrm{x}$ increase in power. If $100 \mu \mathrm{N}$ total thrust was necessary for a given propulsion configuration, arrays of 2600 emitters would be required. The total power consumption, increased to $2.5 \mathrm{~W}$, is well within the range that can be provided by even a single unit CubeSat. With the currently achieved packing density $\left(213\right.$ emitter $\left.\mathrm{cm}^{-2}\right)$, this array would require approximately $12 \mathrm{~cm}^{2}$ of surface, still just $12 \%$ of a CubeSat face.

\section{Conclusion}

Due to their high achievable specific impulse, efficiency and miniaturizability, microfabricated electrospray thrusters are one of the most promising technologies for the propulsion of small spacecraft. The inclusion of integrated acceleration electrodes is a key step in their development, allowing not only significant performance increases but also a number of new device features.

Acceleration improves the thrust and specific impulse of the device not only by raising the energy of the emitted particles, but also by increasing the thruster efficiency. Models of the acceleration electrode indicate that, within the design space of interest, the effect of these electrodes on the extraction of the spray is negligible, an element confirmed by experiments. This decoupling is critical as it permits tuning of the acceleration potential while keeping an optimal extraction voltage.

We developed a process with allows the wafer-level fabrication of large arrays of emitters, each with integrated extractor and accelerator electrodes. The emitter's critical dimensions are highly repeatable, with carefully isotropically etched tips and high aspect-ratio interiors $(7.9 \pm 0.5 \mu \mathrm{m}$ inner diameter, $100 \mu \mathrm{m}$ deep). The electrodes, insulated by $250 \mu \mathrm{m}$ of glass patterned by a novel micro-sandblasting process, are wafer-level bonded to the emitters to improve device reliability and minimize the footprint.

Arrays of 127 capillary emitters were successfully tested using the ionic liquid EMI- $\mathrm{BF}_{4}$ and presented beams composed of $95 \%$ ions, with current levels in the range of $0.25 \mu \mathrm{A}$ per emitter. From plume shape measurements, we confirmed that the individual accelerator electrodes could effectively focus the plume, bringing the beam half angle from $30^{\circ}$ to $15^{\circ}$.

Next steps will include a further reduction of the emitter inner diameters, with the aim of lowering flow rates and increasing ion 
content. In this way, we hope to achieve purely ionic emission, maximizing the specific impulse and efficiency of the thruster.

\section{Acknowledgments}

This work has been partially supported by the MicroThrust project, grant agreement number 263035, funded by the EC Seventh Framework Programme theme FP7-SPACE-2010, the Swiss National Science Foundation grant 200021-146365 and an ESA NPI contract 4000109063/13/NL/PA. The authors would like to thank project partners TNO, SystematIC, Nanospace and the Swiss Space Center.

\section{References}

[1] Meyer M, Johnson, Palaszewski L, Goebel D M and Coote D 2012 In-space Propulsion Systems Roadmap: Technology Area 02 (NASA)

[2] Spence D, Ehrbar E, Rosenblad N, Demmons N, Roy T, Hoffman S, Williams D, Hruby V and Tocci C 2013 Electrospray propulsion systems for small satellites Proc. 27th AIAA/USU Conf. on Small Satellites 1-7

[3] Marcuccio S, Pergola P and Giusti N 2012 IL-Feep: a simplified, low cost electric thruster for micro- and nanosatellites Proc. ESA-CNES Small Satt. Sys. and Services Symp. (Portorose, Slovenia)

[4] Taylor G 1964 Disintegration of water drops in an electric field Proc. R. Soc. Lond. $280383-97$

[5] Gamero-Castaño M and De La Mora J F 2000 Direct measurement of ion evaporation kinetics from electrified liquid surfaces J. Chem. Phys. 113815

[6] Gassend , B L P Velasquez-Garcia L F, Akinwande A I and Martínez-Sánchez M 2009 A microfabricated planar electrospray array ionic liquid ion source with integrated extractor J. Microelectromech. Syst. 18 679-94

[7] Courtney D G, Li H Q and Lozano P C 2012 Emission measurements from planar arrays of porous ionic liquid ion sources J. Phys. D: Appl. Phys. 45485203

[8] Deng W, Klemic J F, Li X, Reed M A and Gomez A 2006 Increase of electrospray throughput using multiplexed microfabricated sources for the scalable generation of monodisperse droplets J. Aerosol Sci. 37 696-714

[9] Lenguito G, De La Mora J F and Gomez A 2010 Multiplexed electrospray for space propulsion applications Proc. 46th AIAA/ASME/SAE/ASEE Joint Propulsion Conf. and Exhibit (Nashville, Jul. 2010)

[10] Larriba C, Castro S, De La Mora J F and Lozano P C 2007 Monoenergetic source of kilodalton ions from Taylor cones of ionic liquids J. Appl. Phys. 101084303

[11] Legge Jr R S and Lozano P C 2011 Electrospray propulsion based on emitters microfabricated in porous metals $J$. Propul. Power 27 485-9

[12] Romero I, Bocanegra R and De La Mora J F 2003 Source of heavy molecular ions based on Taylor cones of ionic liquids operating in the pure ion evaporation regime J. Appl. Phys. 943599

[13] Krpoun R, Smith K L, Stark J P W and Shea H R 2009 Tailoring the hydraulic impedance of out-of-plane micromachined electrospray sources with integrated electrodes Appl. Phys. Lett. 94163502

[14] Krpoun R and Shea H R 2009 J. Micromech. Microeng. 19045019

[15] Ç Ataman, Dandavino S and Shea H R 2012 Waferlevel integrated electrospray emitters for a pumpless microthruster system operation in high efficiency ion-mode Proc. IEEE MEMS (Paris, Jan. 2012)
[16] Lozano P C and Martínez-Sánchez M 2005 Efficiency estimation of EMI-BF 4 ionic liquid electrospray thrusters Proc. 41 st AIAA/ASME/SAE/ASEE Joint Propulsion Conf. and Exhibit (Tucson, Jul. 2005) pp 1-7

[17] Visee R, de Jong M and Timmerman J 2013 Miniaturized HV power supply Proc. 33rd Int. Electric Propulsion Conf. (Washington, Oct. 2013) pp 1-8

[18] Lozano P C and Martínez-Sánchez M 2004 Ionic liquid ion sources: suppression of electrochemical reactions using voltage alternation J. Colloid Interface Sci. 280 149-54

[19] Gassend B L P 2007 A fully microfabricated two-dimensional electrospray array with applications to space propulsion by Blaise Laurent Patrick Gassend PhD Thesis Massachusetts Institute of Technology

[20] Lozano P C 2006 Energy properties of an EMI-Im ionic liquid ion source J. Phys. D: Appl. Phys. 39 126-34

[21] Fedkiw T P and Lozano P C 2009 Development and characterization of an iodine field emission ion source for focused ion beam applications J. Vac. Sci. Technol. B: Microelectron. Nanometer Struct. 272648

[22] Lozano P C and Martínez -Sánchez M 2005 Ionic liquid ion sources: characterization of externally wetted emitters J. Colloid Interface Sci. 282 415-21

[23] Miller S W, Prince B D and Rovey J L 2012 Capillary extraction of the ionic liquid [Bmim][DCA] for variable flow rate operations Proc. 48th AIAA/ASME/SAE/ASEE Joint Propulsion Conf. and Exhibit (Atlanta, Aug. 2012) pp 1-3

[24] Chakraborty S, Ataman Ç, Dandavino S and Shea H R 2012 Microfabrication of an electrospray thruster for small spacecraft Proc. Power MEMS (Atlanta, Dec. 2012)

[25] De La Mora J F, Navascues J and Fernandez F 1990 Generation of submicron mondisperse aerosols in electrosprays J. Aerosol Sci. 21 673-6

[26] Krpoun R and Shea H R 2008 A method to determine the onset voltage of single and arrays of electrospray emitters $J$. Appl. Phys. 104064511

[27] Lenguito G and Gomez A 2013 Pressure-driven operation of microfabricated multiplexed electroSprays of ionic liquid solutions for space propulsion applications $J$. Microelectromech. Syst. 23 689-98

[28] Ergun S 1952 Fluid flow through packed columns Chem. Eng. Prog. 48 89-94

[29] Martino W, De La Mora J F, Yoshida Y, Saito G and J 2006 Surface tension measurements of highly conducting ionic liquids Green Chem. 8390

[30] Thadani M C 1966 Variation of local void fraction in randomly packed beds of equal spheres Indust. Eng. Chem. 5 265-8

[31] Goodling J S, Vachon R I, Stelpflug W S, Ying S J and Khader M S 1983 Radial porosity distribution in cylindrical beds packed with spheres Powder Technol. 35 23-9

[32] Auwerda G J and Kloosterman J L 2010 Comparison of experiments and calculations of void fraction distributions in randomly stacked pebble beds Proc. Adv. in Reactor Phys. to Power the Nucl. Renaissance (Pittsburgh, May 2010)

[33] Pawlowski A G, Belloy E, Sayah A and Gijs M 2003 Powder blasting patterning technology for microfabrication of complex suspended structures in glass Microelectron. Eng. $68557-65$

[34] Spierings G 1993 Wet chemical etching of silicate glasses in hydrofluoric acid based solutions J. Mater. Sci. 28 6261-73

[35] Lenard R X, Kraitz S H, Nogan J and Schmidt C 2007 Progress in MEMS-based field emission thrusters at Sandia national laboratories Proc. 43rd AIAA/ASME/SAE/ASEE Joint Propulsion Conf. and Exhibit (Cincinnati, Jul. 2007)

[36] Ryan C et al 2013 Experimental progress towards the MicroThrust MEMS electrospray electric propulsion system Proc. 33rd Int. Electric Propulsion Conf. (Washington, Oct. 2013) 